\title{
Assessment of Dental Anxiety Levels among Students of Medical and Dental Sciences
}

\section{Ambrina Qureshi ${ }^{1}$}

Naghma Azad ${ }^{2}$

Dur-e-Sameen $^{3}$

Maham Baqai $^{4}$
BDS, MPhil

BDS, MCPS

MBBS, MPhil

BDS

\begin{abstract}
:
Objective: This study was designed to look in the subjective ratings of dental anxiety levels among health care students (both dental and medical students) of a public sector University hospital and explore the difference in terms of their age, gender and their health related field of study.

Methodology: A cross sectional study was conducted on medical and dental students of Dow University of Health Sciences, Karachi $(n=315)$. Dental anxiety level of the study participants was evaluated by using Modified Dental Anxiety Scale (MDAS).

Results: Two hundred ( $\mathrm{n}=200)$ participants completed the questionnaire with $63.5 \%$ response rate. Out of the total $59 \%$ belonged to dental whereas $41 \%$ belonged to medical field. Only $3 \%$ of the total was observed to have mean anxiety score of $<5$. On the other hand majority $(90 \%)$ suffered from slight-to-fair anxiety and only $7 \%$ were observed as dental phobic (extreme anxiety). Statistically significant differences $(\mathrm{p}<0.05)$ in ranked mean anxiety scores were observed according to gender, age group and field of study differences respectively.

Conclusion: It was concluded from the present study that female dental students of age group $\leq 21$ years scored higher dental anxiety scores than medical male counter group of same age group. Particularly females were found to be more fearful of local anesthesia injection.
\end{abstract}

KEYWORDS: Dental Anxiety, Medical students, Dental students.

HOW TO CITE: Qureshi A, Azad N, Dur-e-Sameen, Baqai M. Assessment of Dental Anxiety Levels among Students of Medical and Dental Sciences. J Pak Dent Assoc 2017; 26(2): 54-58

Received: 1 May 2017, Accepted: 15 June 2017

\section{INTRODUCTION}

A Although 'dental anxiety' and 'dental phobia' are the terms interchangeably used, but based on their population based prevalence it must be noted that

1. Professor and Head, Department of Community Dentistry, Dr. Ishrat U1 Ebad Khan Institute of Oral Health Sciences, Dow University of Health Sciences, Karachi, Pakistan.

2. Assistant Professor, Department of Oral Medicine/ Oral Diagnosis, Dr. Ishrat-Ul-Ebad Khan Institute of Oral Health Sciences, Dow University of Health Sciences, Karachi, Pakistan.

3. Assistant Professor, Department of Pathology, Dow International Medical College, Dow University of Health Sciences, Karachi, Pakistan.

4. Lecturer, Sir Syed College of Medical Sciences, Karachi, Pakistan.

Corresponding author: "Dr. Ambrina Qureshi"

<ambrina.qureshi@duhs.edu.pk> there may be a difference between the two terms. Researchers have found that dental anxiety is more prevalent $(20 \%)$ among general population than dental phobia $(5 \%){ }^{1}$. When it comes to any kind of advice in terms of dental problems being followed by the patient, it becomes the worst barrier for the dental care professionals. By definition dental anxiety is known as fear of visiting a dentist for any sort of dental procedures; whereas phobia is an extreme level of fear or anxiety. The presence of elevated levels of such an anxiety or fear amongst patients brings about negativity towards dental treatment that could be accomplished efficaciously otherwise. In fact dental anxiety is a major factor responsible for posing a major threat to oral health and due to this the patient can suffer from multiple serious complications like, septicemia, osteomyelitis of face and sinusitis $^{2}$. 
The most nerve-wracking situation in tackling an anxious patient might occur if confronted by a health care student. A recent local study using Depression-Anxiety-Stress Scale (DASS-21) found that dental students and that too females are found to be considered as more panicky, touchy and nervous as compared to male dental students ${ }^{3}$. Although DASS-21 is more commonly used by oral health researchers due to its ability to assess wide spectrum of anxiety, yet Corah Dental Anxiety and its modified version Modified Dental Anxiety Scale (MDAS) has been observed to be more inclusive, more valid and consistent, with much simpler and stable responding system among University level students ${ }^{4}$.

What so ever the dentists may experience in their practice while confronted by an anxious patient, yet very little is known about the level of anxiety among health care students when they confront a dental visit themselves. Adequacy of knowledge about the dental procedures may exacerbate anxiety making health care students more cautious about their own dental treatment. Hence this study was conducted to report self-responded assessments of dental anxiety levels among health care students (both dental and medical students) of a public sector University hospital and explore the difference in terms of their age, gender and their health related field of study.

\section{METHODOLOGY}

All medical and dental students of Dow University of Health Sciences were aimed to be approached and MDAS questionnaire was distributed to be filled by them. This included five items with simplified 5-point scale responding system ranging from "not anxious" to "extremely anxious". The five multiple-choice items were:

"If you went to your dentist for treatment tomorrow, how would you feel?"

"If you were sitting in the waiting room, how would you feel?"

"If you were about to have a tooth drilled, how would you feel?"

"If you were about to have your teeth scaled and polished, how would you feel?"

"If you were about to have a local anesthetic injection in your gum, how would you feel?"

The responses of all five items were added up to project a sum of dental anxiety scores, where score 6-18 indicated slight-to-fairly anxious and score of 19-25 indicated extremely anxious or possibly dental phobia.

JPDA Vol. 26 No. 02 Apr-Jun 2017
The students were clarified about the questions used in study tool prior distribution. Only five hundred questionnaires could be conveniently distributed and participants were asked to return back on the same day. Data was entered and analyzed using statistical software Stata 11.0. Descriptive statistics included means (standard deviation) of participants' age and frequency distribution for their gender and health field the students belonged to. Overall prevalence of dental anxiety and dental phobia were also calculated. Inter-group comparisons were analyzed using Mann Whitney Test as none of the grouping variables (gender (male/female), age $(\leq 21 />21$ years) and field of study (dental students/ medical) fulfilled the normality assumption. Statistical significance was assessed on the probability values of $\mathrm{p}<0.05$ and confidence interval of $95 \%$.

\section{RESULTS}

Despite of aiming at all medical and dental students to be approached, only 315 were easily accessible and were recruited. Two hundred $(n=200)$ participants returned their questionnaire without any missing information; rest either did not return or were incompletely filled giving the reason that they have never visited a dentist for any kind of treatment. Hence information of only 200 questionnaires was entered as final data considering $63.5 \%$ response rate. Out of the total students included $59 \%$ belonged to dental field of study whereas $41 \%$ belonged to medical field. Gender-vice distribution was 68\% females and 32\% males. The mean age of participants was 21.57 years (minimum=18 years, maximum $=25$ years).

The overall prevalence of anxiety categories in relation to gender and field of study is displayed as Graph 1, with an overall mean anxiety score of 12.08 (minimum=5, maximum=21). Only $3 \%$ of the total was observed to have mean anxiety score of $<5$. On the other hand majority $(90 \%)$ suffered from slight to fairly anxious group and only $7 \%$ were observed as dental phobic (extreme anxiety). Statistically significant differences $(p<0.05)$ in ranked mean anxiety scores were observed according to gender, age group and field of study differences respectively as can be seen in Table 1.

\section{DISCUSSION}

The current study aimed at not only assessing the prevalence of anxiety among medical and dental students but also aimed at observing any difference in the anxiety level in terms of gender, age and field of study of the target participants. The study found that majority of students 


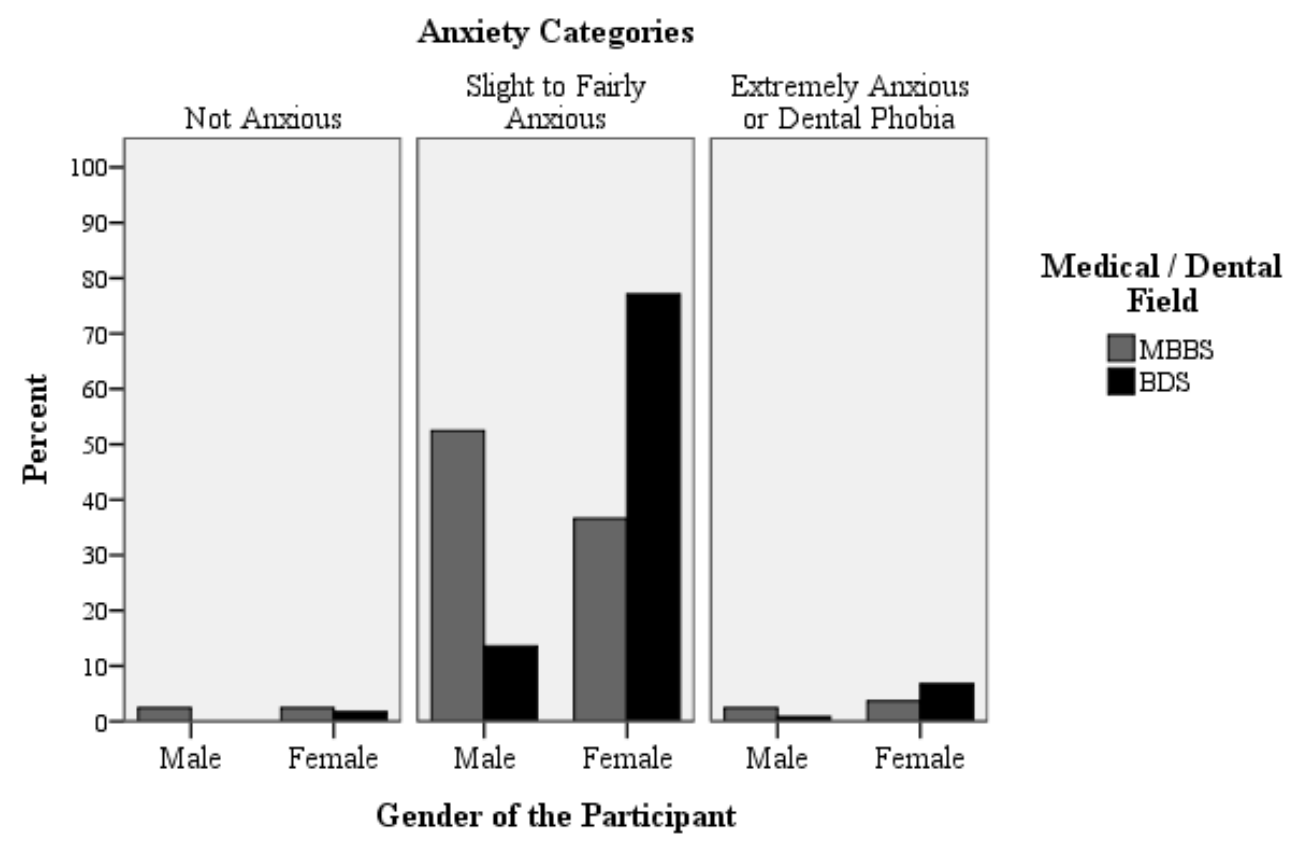

Graph 1: Prevalence of Anxiety Categories according to Field of Study and Gender of Students (n=200).

Table 1: Difference in Ranked Mean Anxiety Scores According to Grouping Variables

\begin{tabular}{|c|c|c|c|c|}
\hline \multicolumn{2}{|c|}{ Grouping Variables } & $\mathrm{n}=200$ & Mean Rank & $\mathrm{p}$-value (2-tailed) \\
\hline \hline \multirow{2}{*}{ Gender } & Male & 64 & 88.01 & $0.036^{*}$ \\
\cline { 2 - 5 } & Female & 136 & 106.38 & \\
\hline \multirow{2}{*}{ Age } & $\leq 21$ years & 96 & 108.64 & 0.055 \\
\cline { 2 - 5 } & $>21$ years & 104 & 92.99 & \multirow{2}{*}{$0.021^{*}$} \\
\hline \multirow{2}{*}{ Field of Study } & MBBS Students & 82 & 89.21 & 108.34 \\
\hline
\end{tabular}

*Statistical significance $\mathrm{p}<0.05$.

despite of gender, age and field of their study suffered from a range of slight-to-fair anxiety level. Moreover, 7\% of these students were also found to suffer from dental phobia; which is almost the same as globally found among general population, that is $5 \%{ }^{1}$. It is worth mentioning that similar studies conducted to assess dental anxiety have different cutoff values for dental anxiety levels, depending upon the number of questions included. Some studies considered anxiety cut-off score of 15 where above 15 considered as high ${ }^{5-7}$ or even severe anxiety level ${ }^{8,9}$ and others considered 19 and above as high and severely high ${ }^{10,11}$.

When closely observed female BDS students were found more prevalent as compared to male MBBS students to be suffering both from anxiety as well as dental phobia. Similar result was reported in other study ${ }^{9}$. This finding was unlike to that reported in a similar study where medical students reported dental anxiety with mean score $>15^{12}$. Likewise, another similar study found dental students less anxious than medical and pharmacy students ${ }^{5}$. Self-reported anxiety level among female BDS students were found to be fairly raised for the two pertinent questions, that is related to " tooth drilled" and "local anesthetic injection" The reason that BDS students were observed with raised dental anxiety score could be probably due to their exposure to prior knowledge regarding how these two procedures are routinely performed. The lack of knowledge about dentistry among medical students might be the reason for lower anxiety scores as they do not know sufficiently about the procedures 
performed in dentistry. Female gender found with increased dental anxiety was in compliance with other similar crosssectional ${ }^{8}$ and longitudinal studies ${ }^{12}$. The reason suggested by one of these studies was neuroticism; similar to what an earlier study on the same study group was conducted to identify anxiety spectrum factors through factor analysis, attributing these females as more "touchy", "panicky" and "nervous" than males ${ }^{3}$. Females related to health care studies are generally found to be more tensed ${ }^{13}$.

The reason for considering age 21 years as a cut-off to assess its relationship with dental anxiety in this study was because usually medical and dental students are either in their final year of studies or have already cleared their final year. The result of this study showed that students of age 21 years and below were found with higher anxiety score as compared to those belong to age group $>21$ years. However, this difference was slightly significant which may be attributed to a chance finding. Although this finding was similar to what found in other studies, where fresher and sophomores were found more anxious than senior students 11,14

The differences in all variables were noticeably large and of statistical significance, except for that attributed to age where marginal difference was observed. However, the results must be critically considered in terms of significant differences observed between groups related to mean ranked dental anxiety scores or individual items score of anxiety scale which probably could be misrepresentative and possibly reflect a difference by chance and not of an actual clinical significance. This could be attributed to small sample size and did not represent the general population, probably compromising the power of the study.

\section{CONCLUSION}

It was concluded from the present study that female dental students of age group $\leq 21$ years scored higher dental anxiety scores than medical male counter group of same age group. Particularly females were found to be more fearful of local anesthesia injection.

\section{RECOMMENDATIONS}

Although the results based on absolute statistical significance must be inferred with care, still it is suggested that dental anxiety and dental phobia may have an adverse effect ones quality of life. Hence appropriate measures must be taken to alleviate the obstacles related to the factors that may be related to the determinants of dental anxiety and fear. Dentists must be trained particularly in communicating and psychologically managing ${ }^{15,16}$ their patients prior any therapy no matter he/she is treating his/her dentist fellow or a dental student. Dentists must understand that patient is after all a patient no matter he/ she belongs to a health related field or not.

\section{ACKNOLEDGEMENT}

We appreciate the time and effort put in by the students of Dow University of Health Sciences to participate in this study.

\section{FINANCIAL INTEREST}

None.

\section{CONFLICT OF INTEREST}

None.

\section{AUTHORS' CONTRIBUTION}

Complete manuscript writing was performed by the first author (AQ); however the research idea was conceived by fourth author (MB). Data was collected and recorded by last three authors (NA, DS and MB). Statistical analysis was performed by the first author (AQ). All authors approved the final version and agreed to be responsible for accuracy of results and integrity of the research.

\section{REFERENCES}

1. Wide BU, Carlsson V, Westin M, Hakeberg M. Psychological treatment of dental anxiety among adults: a systematic review. Eur J Oral Sci 2013; 121: 225-234.

2. Sanikop S, Agrawal P, Patil S. Relationship between dental anxiety and pain perception during scaling. J Oral Sci 2011; 53 (3):341-348

3. Qureshi A, Haider MM, Khan R. Exploring Gender Distribution in Anxiety Spectrum through Factor Analysis among Dental Students. J Pak Dent Assoc 2016; 25 (03): 87-92

4. Shaikh MA, Kamal A. Over Dental Anxiety Problems Among University Students: Perspective from Pakistan. J Col Phy Surg Pak 2011; 21(4): 237238.

5. Hantash R O Abu, Younis M H Abu, Aker M M. Dental anxiety and fear among medical field students at Al Quds University. Br J Med Med Res 2014; 4(12): 2312-2321. 
6. Donka G. Kirova. Dental anxiety among dental students. JAMAB-Annual Proceeding (Scientific Papers) 2011; 17(2): 137-139.

7. Al-Omari WM, Al-Omiri MK. Dental anxiety among university students and its correlation with their field of study. J Appl Oral Sci 2009.

8. Sghaireen MG, Zwiri AMA, Alzoubi IA, Qodceith SM, Al-Omiri MK. Anxiety due to dental treatment and procedures among university students and its correlation with their gender and field of study. Int $\mathbf{J}$ Dent 2013. Article ID 647436, 5 pages. doi:10.1155/2013/647436

9. Hakim H, Razak IA. Dental Fear among Medical and Dental Undergraduates. Sc World J 2014; Article ID 747508, 5 pages. doi:10.1155/2014/747508

10. Gunjal S, Pateel DGS, Parkar S. Dental Anxiety among Medical and Paramedical Undergraduate Students of Malaysia. Int J Dent 2017; 2017:4762576. doi:10.1155/2017/4762576

11. Storjord HP, Teodorsen MM, Bergdahl J, Wynn R, Johnsen J-AK. Dental anxiety: a comparison of students of dentistry, biology, and psychology. J Multi Health 2014; 7:413-418. doi:10.2147/JMDH.S69178.
12. Peretz B, Mann J. Dental anxiety among Israeli dental students: A four-year longitudinal study. Eur J Dent Edu 2000; 4(3): 133-137

13. Fontes de Oliva CE, Vieira Rocha MM, Rodrigues de Santos AT, Vieira de Melo E, Martins LAN, Andrade TM. Common mental disorders and associated factors among final-year healthcare students. Revista da Associacao Medica Brasileira [Internet] 2014; 60( 6 ): $525-530$

14. Ali S, Farooq I, Khan SQ, Moheet IA, Al-Jandan BA, Al-Khalifa KS. Self-reported anxiety of dental procedures among dental students and its relation to gender and level of education. J Taib Univ Med Sc 2015; 10(4): 449-453.

15. Armfield JM, Heaton LJ. Management of fear and anxiety in the dental clinic: a review. Aust Dent J 2013; 58(4):390-407. [PubMed]

16. Appukuttan DP. Strategies to manage patients with dental anxiety and dental phobia: literature review. Clin Cosm Invest Dent 2016; 8:35-50. doi:10.2147/CCIDE.S63626. 